\title{
FUENTES DOCUMENTALES PARA EL ESTUDIO DE LA PROSTITUCIÓN EN LOS SIGLOS XVI Y XVII EN EL ARCHIVO Y BIBLIOTECA DE LA UNIVERSIDAD DE SALAMANCA
}

\author{
Agustín Vivas Moreno* y Luis Arias González**
}

En torno al papel desempeñado por la mujer en la Edad Moderna hispana, hay una serie de tópicos que no por muy repetidos resultan, en este caso, forzosamente falsos ${ }^{1}$, sino más bien lo contrario; empezando, por ejemplo, por el puesto secundario que ocupaba en el entramado social, siguiendo por la nula actuación en el ámbito de la alta economía, las instituciones políticas, culturales, etc., y terminando con la práctica ausencia de figuras de importancia, salvo las consabidas individualidades (reinas, nobles o religiosas...). Sin embargo, quedan muchos aspectos casi desconocidos sobre la mujer, especialmente en lo que se refiere a aquellas facetas inmateriales, con pocas posibilidades de ser reducidas a números, como sucede con la visión moral que de ella se tenía o se pretendía imponer, campo éste en el que encuadramos nuestro trabajo.

El concepto moral presente en este tiempo, viene definido -como casi todos los que se han dado en la historia de la Humanidad-por la Religión. A partir del Concilio de Trento, la Contrarreforma se basará en una fijación y reglamentación de todo lo moral en el mundo católico, con sus normas -leyes y

* Prof. Fac. Biblioteconomía y Documentación Univ. de Extremadura.

** Profesor Instituto

1. Vid. VIGIL, M.: La vida de las mujeres en los siglos XVI y XVII, Madrid, 1986; FERNÉNDEZ VARGAS, V. y LÓPEZ-CORDÓN, $M^{\text {a }}$ V.: "Mujer y régimen jurídico en el Antiguo Régimen: una realidad disociada" en Ordenamiento jurídico y realidad social de las mujeres. Cuartas jornadas de investigación interdisciplinaria, Madrid, 1986. En la misma publicación: FRIEDMAN, E.-G.: "EI estatus jurídico de la mujer castellana durante el Antiguo Régimen"; CAPEL, R.M"a: "Los protocolos notariales y la historia de la mujer en la España del Antiguo Régimen". También AMELANG, J. y NASH, M.(eds.): Historia y género. Las mujeres en la Europa moderna y contemporánea, Valencia, 1990; LUNA, L.S. (eds.): Mujeres y sociedad. Nuevos enfoques teóricos y metodológicos, Barcelona, 1991; ANDERSON, B.S. y ZINSSER, J.P.: Historia de las mujeres: una historia propia, Barcelona, 1991, 2 vols (el caso español, en Apéndice, pp. 583-668, vol. 2). 
decretos conciliares- $y$ las posibles transgresiones a las mismas. ${ }^{2}$ Trento no es que crease absolutamente todo "ex nuovo", puesto que recogió un fuerte componente heredado de las épocas anteriores y que provenía tanto del campo puramente religioso, como del filosófico y -el muy importante- de la tradición cultural. Toda esta amalgama, es la que traza las pautas de consideración sobre la mujer y la moral ${ }^{3}$, que fue plenamente aceptada en España entre otras cosas porque ya se venía haciendo. Según la posición y el estado social desempeñado, variaban las responsabilidades éticas y los peligros morales consiguientes; conviene aclarar que esta preocupación tipológica y clasificatoria a la hora de enjuiciar moralmente, afectaba a todas las capas de la población, agrupadas por edad, oficios, condición y hasta raza. ${ }^{4}$ Volviendo al caso de la mujer:

1. Hay un primer gran apartado -las seglares- en el que se incluyen, por este orden, las doncellas, las casadas y las viudas;

2. un segundo grupo -las religiosas-, con una jerarquización mucho más marcada, integrado por las beatas, novicias, monjas y preladas.

Sin embargo, por encima de esta división social con sus repercusiones morales y su correspondiente casuística, existían unos convencionalismos no sólo religiosos, que se hacían extensibles a los dos conjuntos y que podemos reducir en estas dos afirmaciones:

1. la mujer es una persona frágil por naturaleza, proclive a todo tipo de debilidades ${ }^{5}$ y abocada más al pecado que el varón. De esta forma, la respon-

2. LE PLAT, S.: Monumentorum ad Concil. Trident. amplissima collectio, Lovaina, 1781, 7 vols. Vid. la interpretación que efectúa DUPRONT, A.: "le Concile de Trente" en Le concile et les conciles, Chevetogne, 19660, pp. 195-243.

3. Estos temas fueron especialmente tratados en la Tercera etapa del Concilio, que va desde Enero de 1562 a Diociembre de 1563, bajo el Pontificado de Pio IV (Vid. SOSTA, S.: Die römanische Kurie und das Konzil von Trient unter Pius IV, 1904-1914, 4 vols.).

4. Para constatarlo basta simplemente con hojear alguno de los múltiples manuales de confesión del momento, por ejemplo para la agrupación por oficios el de Juan López de Segura (Confessionario assi útil para los confessores y para saberse los penitentes examinar y confessar.......], Burgos, Juan Junta, 1555), sobre grupos de ediad el del jesuita Andrés Mendo (Instrucción breve de examinar la conciencia para confesión general o particular con adiciones especiales para los estudiantes de la Universidad de Salamanca, Salamanca, s.i., s.f.) y sobre grupos raciales podemos señalar los más conocidos dedicados a los indígenas como el del franciscano Alonso de Medina (Conffesionario M; ayor en la lengua mexicana y castellana[...], México, Pedro Balli, 1578; Para un análisis de este confesionario, ver nuestro trabajo, VIVAS MORENO, A. y ARIAS GONZÁLEZ, L.: "LOs manuales de confesión para indígenas del s. XVI. Hacia un nuevo modelo de formación de la conciencia" en Studia Histórica, Vol. XI, 1993, Salamanca, 1993, pp.245-260).

5. La justificación de la debilidad femenina se daba hasta en el momento de la concepción, puesto que la doctrina tradicional afirmaba que la entrada del alma en el feto se producía a los cuaremnta días en el caso del varón y a los ochenta en el de la mujer (Juan Machado: Perfecto confesor i cura de almas [...], Barcelona, Pedro Lacavallería, 1641). La alusión constante a esta fragilidad en los sermones y misiones será una práctica común muy lamentada posteriormente por Feijoo (Cartas Eruditas, tomo V, carta $5^{a}$ ). 
sabilidad de sus actos no era total, lo que en cierto modo estaba justificando la necesidad de una tutela permanente del hombre hacia ella; ${ }^{6}$

2. al lado de esta protección constante concebida casi como una minoría de edad indefinida, de la que no se escapaban ni las viudas ${ }^{7}$, se procuró una separación total de los dos sexos. La cercanía física de hombres y mujeres aparece como un constante peligro y ocasión de pecar, daba igual que el lugar fuera una plaza, el teatro, el mercado ${ }^{8}$ o las iglesias, conventos ${ }^{9}$ y hasta los mismos confesionarios..$^{10}$ Este aislamiento procuró preservar tanto de una supuesta lujuria natural como, sobre todo, del deshonor familiar que iba vinculado a la integridad de sus mujeres."

De todo esto, parecería que queda totalmente al margen un tipo femenino de difícil encuadramiento, y que en cierto modo es una consecuencia de los dos anteriores y hasta contribuye, quizá sin saberlo, a su sostenimiento. Nos referimos a la figura de la prostituta, tan presente en la literatura hispánica $y, s$ in embargo, tan poco estudiada si exceptuamos los aspectos pintorescos y ane cdóticos ${ }^{12}$ de los que procuramos huir, ya que nuestro interés es el enfoque moral

6. El control de padre, hermanos y marido, propio del estado seglar se continuaba en el religioso ejercido por los obispos o por la rama masculina de la orden. (DOMÍNGUEZ ORTIZ, A.: La sociedad española en el siglo XVII, Madrid, 1970, p. 124).

7. La obligada solución era volver a casarse, entrar como servidora en una casa o bien ingresar en beaterior y "emparedamiento" (ORELLANA, M.A.: Tratado histórico apologético de las mujeres emparedadas, Valencia, 1887).

8. El capuchino Corella, predicador real, arremete contra los zapateros que "çalzan a las mugeres" (Práctica de el Confessionario y explicacion de las LXV proposiciones comndenadas por la Santidad de N.S.P. Inocencio XI [...], Madrid, Imprenta Real, 1690).

9. El escrito de Manuel de Vega y Quadrados (Retiro de profanas comunicaciones necesario a las espossas de XPto [...], Toledo, s.i., 1651) recoge todo lo relacionado con las llamadas "devociones de monjas".

10. Los peligros de contacto sexual entre el confesor -"solicitantes torpes" como se les denomina- y el penitente dieron lugar a una literatura abundantísima (por ej. de Rodrigo de Acuña el Tractatus de Confessariis Solicitantibus, Valladolid, Juan de Rueda, 1620), casi toda ella derivada de las reglamentaciones dictadas al respecto por Pio IV -1561-, Clemente VIII -1592- y Gregorio XV -1622-, aunque llegará esta preocupación hasta el s. XVIII con Benedicto XIV.

11. Una aproximación al tema de la valoración social de la virginidad y de la castidad pública, así como su identificación con el honor, es lo que hace MARAVALL, J.A.: Poder, honor y élites en el s. XVII, Madrid, 1979. Sobre el origen histórico -musulmán, sobre todo- de esta obsesión barroca por la virginidad, vid. FLANDRIN, J.L.: La moral sexual de Occidente, Barcelona, 1984; V. RIVELA, Ma M.: "La construcción de lo femenino entre musulmanes, cristianos y judios" en Relaciones entre musulmanes, judios y cristianos, Actas del 17 Congreso Internacional de Ciencias Históricas, Madrid, 1990, e.p.

12. Resulta curioso que el tema de la prostitución en la historiografía española, apenas aparece de forma aislada sino en relación con otros asuntos: literatura, Inquisición, minorías raciales y religiosas, el ordenamiento jurídico, etc. Vid. DELEITO PIÑUELA, J.: La mala vida en la España de Felipe IV, Madrid, 1967; DEFORNEAUX, M.: La vida cotidiana en España en el Siglo de Oro, Barcelona, 1983; la ya citada de VIRGIL, M. etc.: Exceptuamos el estudio de PERRY, 
sobre la misma y no otro. Acercarse a la consideración religiosa que se hizo de la prostitución en los siglos XVI y, especialmente, el XVII, supone enfrentarse a todo un conjunto de escritos tan curiosos como dispersos; es decir, a los Sermonarios, a las obras doctrinarias, los manuales de confesión y a las compilaciones de casos y dudas de conciencia. ${ }^{13}$ La imagen que de ellas se nos da en estas fuentes, sorprende porque no está tan alejada, como podía pensarse, se las restantes mujeres; también se encuentra sujeta a la potestad de un hombre casi siempre ${ }^{14}$, y se vio afectada por una serie de normas para que no pudiera ser confundida, ni se entremezclara libremente ${ }^{15}$. Su oficio recibía la consideración de útil para la República y necesario para el bien común ${ }^{16}$, puesto que de algún modo se aseguraba la preservación de las restantes mujeres, cuya integridad había que mantener. Esto generaba una contradicción moral evidente: el orden político y social, sostenido ideológicamente por el catolicismo amparaba a la prostitución como un mal menor, siendo ésta condenable desde el punto de vista religioso. Cómo se mantuvo esta paradoja ética ${ }^{17}$, qué argumentos se usaron y cuál fue la fórmula de compromiso adoptada, son los aspectos que nos proponemos tratar aquí, aunque antes resulta imprescindible mencionar, aunque sea muy brevemente, las nociones que sobre la sexualidad y el Pecado se

M.E.: "Lost Women in Early Modern Seville: The Politics of Prostitution" en Femenist Studies, Vol. IV, Febrero, 1978, pp. 195-214.

13. Mayoritariamente, hemos consultado los fondos existentes en la Biblioteca Universitaria de Salamanca (B.U.S.A.), como en su Archivo (A.U.S.A.). La procedencia original de much as de estas obras fue el Colegio de la Compañía de Jesús (especialmente en B.U.S.A., Ms. 761 y A.U.S.A., P.V. 109); al ser cerrado por la expulsión del s. XVIII, sus libros fueron depositados en la Universidad (Vid. MARCOS RODRÍGUEZ, F.: "La Biblioteca Univertsitaria salmantina" en AA.VV.: La Universidad de Salamanca /I Doccencia e Investigación, Salamanca, 1990, pp. 28 8289). VIVAS MORENO, A. Fondos documentales del Archivo Histórico de la Universidad de Salamanca. La Colección de Papeles Varios: análisis descriptivo, tesauro y gestión documental automatizada. Tesis doctoral. Salamanca, 1998. 6 vols.

14. No aparece en la Literatura, ni una sola mujer de la vida totalmente libre o desvinculada de un hombre, si siquiera las pícaras más conspicuas e ingeniosas. Ni la Lozana de Delicado (1528), ni la Justina de Francisco López de Ubeda (1605), ni la hija de la Celestina de Salas Barbadillo (1612), ni la Teresa de Manzanares de Castillo Solórzano (1632), son autónomas o buscan crear un contrasistema frente al dominante.

15. Los distintivos y accesorios para señalar a las prostitutas -ramas en las puertas, mantos amarillos, sayas con picos, gorros rojos, etc. - fueron generales a toda Europa y usados desde muy antiguo. (OTIS, L.L.: Prostitution in Medieval Society, Chicago, 1985).

16. En este sentido, elogio sobre el oficio de alcahuete, que Cervantes pone en labios de D. Quijote ( $1^{a}$ parte, cap. XXII), resulta altamente significativo, si es que obviamos la parte de amarga autosátira que encierra, puesto que fue relacionado con esta profesión en su oscuro procesamiento de Valladolid (1603).

17. La paradoja, la contradicción, está en el fundamento del espíritu barroco español; ese "concierto de desconciertos" que Gracián pone en boca de Critilo, hace que el ser se desdoble, falto de unidad, o que Quevedo (La hora de todos y la Fortuna con seso) y Tirso (La República al revés) usan como argumentos principales de su obras. La moral acomodaticia de la que luego hablaremos, no es más que el fruto evidente de esta contradicción. 
daban, puesto que sin ellas no se puede entender el complejo entramado argumental que se montó para sostener la paradoja antes mencionada.

\section{Sexualidad y pecado}

El Pecado, a partir del Concilio tridentino, se define como la transgresión o el incumplimiento de la normativa perfectamente establecida que sigue un modelo de raiz jurídica. Se construyó así una interpretación objetiva, exter ior, jerarquizada y legalista del Pecado frente a la visión individualista, subjetiva y omnipresente de la Reforma Protestante; precisamente el Sacramento de la Penitencia se convirtió en el eje motor de esta nueva moralidad. ${ }^{18}$ Esto no quiere decir que existiera una especial obsesión por los yerros sexuales en la es paña de los siglos XVI y XVII, tal y como algunos autores sostienen. Si analizamos los manuales de confesión y comparamos la parte que dedican al sexto $y$ al noveno mandamientos o a la lujuria en general, con el resto, percibimos que ocupan proporcionalmente menos espacio, puesto que se le presta una atención menor que a los demás, y exactamente lo mismo se constata en los casos de conciencia. Por si esto no fuera suficiente, predicadores, confesores y misioneros insisten una y otra vez en la necesidad de relativizar el tema y no darle demasiada importancia. ${ }^{19}$ Pero tampoco debemos caer en el error contrario, sosteniendo la idea de un pudor intencionado o, mejor dicho, de un puritanismo latente en los círculos moralistas del momento lo que hubiera impedido tratar cualquier tema relacionado con el sexo. Nada más lejos de la realidad; basta con leer el libro del jesuita Tomás Sánchez (De sancto matrimonii sacramento disputationum tomi tres, Genova, 1592), especialmente sus dos últimos libros en los que recoge todas las prácticas sexuales imaginables, con un pormenor y una claridad descriptiva sorprendentes. De una manera u otra, los manuales de confesión publicados con posterioridad al trabajo de Sánchez, se hicieron eco del mismo, lo que nos da una completa idea de su difusión y conocimiento en todos los ámbitos. ${ }^{20}$

Desde un punto de vista sancionador, lo primero que se advierte es que la lascivia femenina se vé de manera mucho más benigna que la masculina. Faltas que en el hombre son consideradas como "delitos enormes y atroces" -la homosexualidad, por ejemplo-, no lo son tanto en el caso de la mujer; una vez más, la fragilidad se su naturaleza le exculpa. Lo que queda bien claro es que

18. LEA, H.: A History of Auricular Confession, Londres, 1896.

19. Afirma Francisco Farfán en la obra que citaremos más adelante que "los pecados espirituales, como la embidia, la soberbia, la infidelidad, etc., son de suyo más graves que las carnales" y argumenta que lo primero por ser del espíritu que es " más noble que la carne", lo segundo por el objeto y lo tercero por el propio motivo de la falta.

20. Vid. sobre este escrito las reflexiones que realiza CARO BAROJA, J.:. "Un ejemplo famoso" en Las formas complejas de la vida religiosa (Siglos XVI y XVII), Madrid, 1985, pp. 301-305. 
ella debe actuar siempre como un elemento pasivo y receptor -identificación reiterada hasta la saciedad con el término "vaso"-; cualquier alteración de este rol es lo que resultaba verdaderamente pecaminoso. Por eso, la prostituta no se considera una pecadora superlativa, incapaz de salvarse ${ }^{21}$, puesto que al fin $y$ al cabo era un mero instrumento final del Pecado sin más ${ }^{22}$; la verdadera culpabilidad la tenían aquellas que fomentaban activamente las apetencias del varón mediante conversaciones, afeites o vestidos ${ }^{23}$.

Lo segundo que apreciamos es la clasificación que se da de mayor a menor en torno a estas faltas. La gravedad de las mismas guarda una relación directa con el estado y la condición de la mujer; así, es distinto si ella consiente o si es forzada, si es virgen, si es religiosa, si está casada o no, etc. El otro agravante fundamental lo constituía la circunstancia de truncar la posible procreación a la que debía estar abierta toda actividad sexual. El listado de pecados que se constituyó y su graduación, ya ha sido suficientemente analizado tanto por Delameau para Francia, como por Bartolomé Clavero para el área hispana ${ }^{24}$. En la cúspide de la misma se sitúan los "delitos enormes y atroces" y "contra natura" (bestialidad, sodomía, masturbación, coito interrupto, etc.), junto con el incesto, el estrupo y la violación con todas sus variantes; a continuación vendría el adulterio, también con su escala correspondiente; terminando con un pecado que si no llegaba a estimarse totalmente como venial, sí que era visto como algo menor, nos referimos a lo que entonces se llamaba la "simple fornicación" y dentro de la cuál la prostitución ocupaba el último puesto; además era considerada como una actividad a la que recurría únicamente la capa más baja de la población ${ }^{25}$. Precisamente esta permisividad moral hacia la prostitu-

21. La preocupación cristiana por la salvación espiritual de las prostitutas arranca del S. VII, Justiniano y Teodora fundaron el primer convento para prostitutas; en 1227, el papa Gregorio IX, autorizó la orden de Santa María Magdalena con esta finalidad; en 1520, el papa León X autorizó la regla agustiniana de las "Convertite". Eran relativamente frecuentes los ejercicios, misiones y sermones para ellas, especialmente por parte de los jesuitas -Casa de Santa Marta-.

22. Vid. del Capuchino Fr. Félix de Alamín: Espejo de verdadera y falsa Confession [...], Madrid, Melchor Álvarez, 1695, cap. V, libro 1․

23. La preocupación por el vestir, llegó a hacerse obsesiva tanto en el aspecto económico y suntuario, como en el de la moral sexual. Sólo a título de ejemplo, citamos tres opúsculos entre otros muchos, relativos a este asunto. El de Antonio de León Pinelo: Velos antiguos i modernos en los rostros de las mugeres sus conveniencias i daños[...], Madrid, Juan Sánchez, 1641. El de Dr. Joseph Garcés: La luz más clara que deshaze las tinieblas de la común ignorancia cerca de las opuestas opiniones en la materia de los escotados de las mugeres [...], Jaen, Joseph Copado, 1678. Y, por último, de Fr. Antonio de Ezcaray: Voces del dolor nacidas de la multitud de pecados que se cometen por los trajes profanos afeites[...], Sevilla, Thomás López de Haro, 1691.

24. DELAMEAU, J.: El miedo en Occidente (siglos XIV-XVIII), Madrid, 1989; CLAVERO, B.: "Delito y Pecado. Noción y escala de transgresiones" en Sexo barroco y otras transgresiones premodernas, Madrid, 1990 pp. 57-89.

25. La enumeración de "clientes", hecha por Fr. Gabriel Maqueda sostiene esta consideración: "oficiales, aprendizajes, trabajadores, moçuelos, rufianes, pícaros, esclavos, pages, lacayos, valentones, y otra gente pobre, vil y baxa" (fol. 22v.) 
ción en cierto modo legitimaba también la tolerancia cultura y política hacia la misma. Apenas se dieron ataques frontales ni dialécticos, ni de cualquier otro tipo contra las mancebías o "casas públicas" por parte de los eclesiásticos. La contradicción moral se superaba mediante un silencio tácito y una ignorancia consciente sobre el tema, por otra parte muy poco dado a los lucimientos teológicos o retóricos, lo que hizo que los autores que se decantaron por condenarla fueran escasos y siempre integrados en el grupo de los predicadores menos aplaudidos y de segunda fila, como veremos.

\section{Contra la Prostitución y su legalidad}

Dentro de la Biblioteca y Archivo de la Universidad de Salamanca, hemos hallado dos obras relativas a este problema moral planteado; dos obras escritas en castellano y no en latín, lo que indica que iban dirigidas a un público no excesivamente versado en asuntos doctrinales. Precisamente esto, reafirma una vez más la concepción menor y popular, en el sentido más amplio del térmi$\mathrm{no}^{26}$, que se le pudo dar.

De finales del s. XVI es la publicación de Francisco Farfán a la que tituló: Tres libros contra el pecado de la simple fornicación, donde se averigua que la torpeza entre solteros es pecado mortal [...] y se responde a los engaños de Ios que dicen que no es pecado [...] (Salamanca, Herederos de Mathías Gost, $1585)^{27}$. No se atreve en ella a embestir frontalmente contra la legalidad de la prostitución, aunque sí lo hace de una manera implícita al atacar una de sus principales justificaciones argumentadas. La estructura general de la obra sigue el esquema establecido por los teólogos en sus trabajos con la recurrencia común de las exposiciones y refutaciones, acumulación de citas de autoridades, etc. El primer libro sirve también como introducción doctrinal, ya que comienza fijando el concepto de Pecado con las características propias y clases. Hay en este primer libro una serie de criterios que ejemplifican perfectamente la argumentación usada por Farfán, que era la que trataba de fomentarse ideológicamente entre la población; los principales son éstos:

- se acusa a todos los herejes de ser grandes fornicadores, especialmente a los alumbrados, a Lutero y a su esposa Catalina de la que viene a decir que "por espacio de dos años a cursado el burdel, y vendido su cuerpo a los estudiantes de Vuitemberga";

- aparece un cierto atisbo de independencia religiosa frente al aparato político dominante, de una manera tímida pero que esconde una velada crítica y que se resume en esta frase: "No porque las repúblicas permiten, toleren, o dissimulen muchos peccados mortales, dexan por esso de ser mortales";

26. Vid. GAN JIMÉNEZ, P.: "El sermón y el confesionario, formadores de la conciencia popular" en La Religiosidad popular, II, 1989, pp. 111-124.

27. B.U.S.A. 49974. 
- en el capítulo noveno ("Reprehende el autor a los vulgares, que temerariamente se entremeten a escudriñar $y$ averiguar questiones de Theología"), niega la autoridad de médicos, juristas y hombres de Estado para decidir en este asunto, recalcando la supremacía de la Teología sobre todas las restantes Ciencias.

En el libro segundo ("Donde se prueva por diversas vías en ley natural, divina y humana, que la simple fornicación es peccado mortal"), nos encontramos con la estratificación moral correspondiente de la "simple fornicación"; a la cabeza, como es lógico, se encuentra el amancebamiento permanente entre solteros, le sigue el temporal -"cantoneras" - y termina con el más leve -calificado como "indifferente o vagoroso" - que resulta ser la prostitución, a pesar de lo cual la incluye repetidamente como pecado mortal. A continuación, va toda una completa nómina de heterodoxos que absolvieron la "simple fornicació $\mathrm{n}^{\text {" }}$ (Simón el Mago, Saturnino el Sirio, los Esguiçaros, Adamitas, Bigardos, Vaaaldenses o "pobres de León", Nicolaitas, Durando, Martín de Maestros, Joannes Mayor...) y a la que opone una catarata de citas en contra, extraídas del Antiguo y Nuevo Testamento, del Concilio de Trento, del Derecho Canónico, de Ios Santos Padres y Doctores, incluso de filósofos y poetas del orbe clásico. Por último, en el libro tercero "[...] responde el autor a las apariencias y engaños en que fundan, o pueden fundar su error los que dizen que la simple fornicación no es peccado"; Farfán aprovecha para presentarnos de esta forma las principales justificaciones que se esgrimían para proseguir legitimando la prostitución, paralelizándolas con heterodoxias tales como la predestinación, el panteísmo, el epicureísmo y el regalismo; señalamos las siguientes:

- argumento primero: " porque dicen no poder más y no peca el que haçe lo que no puede evitar"28;

- argumento segundo: "porque conocer carnalmente a una soltera es obra tan natural como lo es miralla, hablalla o comunicalla";

- argumento cuarto:"que las repúblicas [...] disponen y consienten por sus leyes, que aya casas públicas de rameras";

- argumento quinto: dice que no llega a ser pecado si se concierta libremente un precio y se satisface el mismo; ${ }^{29}$

28. A finales del s. XVI y principios del XVII, amplios sectores eclesiásticos, especialmente los franciscanos, seguían viendo al luteranismo confusamente identificado con la doctrina de la predestinación, como una amenaza para la fé católica en España (Vid. la obra de Fray Juan Bautista Fernández titulada Primera parte de las demostraciones Cathólicas, Logroño, 1593).

29. El tema del "Justiprecio" sí que es uno de los temas verdaderamente obsesivos en los manuales de confesión y en los casos y dudas de conciencia; se hacía extensivo a todo tipo de negocios y transacciones (Bartolomé de Medina, O.P.); Breve instrucción de cómo se ha de administrar el Sacramento de la Penitencia[...], Alcalá de Henares, J. de Lequerica, 1589) incluyendo la que aquí se estudia. Vid. el estudio de conjunto que incluye la aportación de los jesuitas, su problemática, etc. hecho por CLAVERO, B.: Usura, del uso económico de la religión en la historia, Madrid, 1984. 
- argumento onceno: algunos médicos "poco christianos" dicen que es malo retener el humor seminal y que se corrompe y produce "melancholias, caymientos, entorpecimientos, opilaciones, calenturas, gota coral $[. . .]^{\prime 30}$

Treinta y siete años más tarde, Fray Gabriel de Maqueda publica su Invectiva en forma de discurso contra el uso de las casas públicas de las mugeres rameras (Granada, Bartolomé de Lorençana, 1622) ) $^{31}$; en buena medida, in spirada en la anterior obra aunque con finalidad mucho más directa. La pretensión del franciscano era la supresión total de los burdeles. Para solicitarla al rey Felipe IV, eleva esta especie de memorial, uno más entre los muchos arbitrios moralistas, con sus mismos planteamientos y estructuras, que proliferaron durante los reinados de Carlos II y de su padre. ${ }^{32}$ Comienza con la argumentación erudita de costumbre, en la que cita ejemplos literarios e históricos del rechazo que egipcios, romanos y bárbaros hicieron de la prostitución; incl uso recoge noticias sobre la inexistencia de este oficio entre los indígenas del Nuevo Mundo ${ }^{33}$ y de Asia. Prosigue con la diatriba fundamental; mientras los defensores de su legalidad echaban mano de las argumentaciones extraídas de los escritos de S. Agustín y Sto. Tomás, según los cuales era un "mal menor" que servía para evitar pecados mucho mayores tales como las violaciones o la sodomía; para contestar, fray Gabriel recurre, paradójicamente como veremos más adelante a la autoridad del jesuita P. Mariana ${ }^{34}$ para así sostener que S. Agu stín se retractó de estas afirmaciones al final de su vida y para puntualizar que el teólogo dominico sólo permitió la tolerancia en tiempos de guerra larga y para uso exclusivo de los soldados. A continuación expone sus seis razones fundamentales para cerrar las casas públicas; razones que entremezclan la utilidad económica y sanitaria, con la ética del pecado y hasta el mismo prestigio político del monarca. Veámoslas:

1. en las casa públicas no sólo no se evita el pecado de sensualidad, sino que se ejercitan y enseñan otros aún peores como las "posturas no naturales", la masturbación y la sodomía;

30. Se atisban es este argumento influencias de la frenología y taxonomía de Huarte de San Juan (Examen de ingenios[...], Baeza, 1575) aunque estuviera prohibida desde 1583.

31. A.U.S.A. P.V. 117

32. En este sentido el asunto que más tinta hizo correr fue, con toda probabilidad, la ilicitud del teatro como espectáculo (Vid. COTARELO Y MORI, E.: Bibliografía de las controversias sobre la licitud del teatro en España, Madrid, 1904).

33. Fray Gabriel se inclina por la corriente espiritual, que considera a los indígenas como seres angelicales anteriores incluso al Pecado Original, y con puntos de contacto con el Cristianismo en aspectos de la Moral Natural e incluso en algunos ritos; Montesinos, Bartolomé de las Casas y casi toda la primera generación de misioneros serían los más conocidos defensores de esta teoría, retomada y llevada a la práctica posteriormente por los jesuitas.

34. No debemos ovidar que esto esconde, quizás sin ser buscado intencionadamente por nuestro autor, una muestra del incico del colapso de la Escolástica ayudado por la labor de los jesuítas, especialmente Vitoria y Suárez. 
2. constituyen un mal ejemplo para los jóvenes y un foco de enfermedades que los "inhabilitan para los estudios de letras y exercicio de las armas y les cortan y acaban los días de la vida";

3. porque en los burdeles se ataca la autoridad de la Iglesia, especialmente por parte de aquellos que sostienen -otra vez la reiteración de siempreque no hay pecado mortal si se paga con justicia a la ramera;

4. en estos sitios se "favorece el vicio y se escarnece la virtud", ya que además en ellos se juega, se bebe, se blasfema, hay riñas, robos...;

5. el tolerarlar va claramente en detrimento de la decencia pública que debe caracterizar al rey de España;

6. que la mayor parte de las prostitutas son heréticas y hechiceras ${ }^{35}$, que aprovechan sus relaciones para extender sus supersticiones y prácticas entre los hombres que las visitan.

Tras estos argumentos y dentro de las más pura línea del arbitrismo ataca, tal y como ya hiciera Farfán, a todos aquellos que sostenían que el varón no puede pasar sin fornicar declarándolos fuera de la ortodoxia. Finaliza apelando de nuevo a Felipe IV, a su obligación de "velar por el bien común", mencionando las medidas restrictivas que supusieron las ordenanzas de Felipe II en $1570^{36}$, el hecho de la inexistencia en Galicia de las mancebías y las "persecuciones populares" contra las rameras que promovieron las predicaciones de S. Vicente Ferrer y Juan de Ávila ${ }^{37}$ a las que apela como último recurso de presión.

Tanto el escrito del s. XVI, como el del s. XVII, a pesar de algún matiz diferenciador poco significativo, debemos encuadrarlos en la corriente moral conocida como "rigorista" contraria a la interpretación más laxa defendida por el "probabilismo". Fueron precisamente los probabilistas, especialmente los jesuitas, quienes contaron con un cada vez mayor poder religioso y ascendencia social, entre otros muchos motivos porque aportaron el sistema moral que permitía al Estado la transgresión de las normas que se aplicaban a los individuos, gracias a la relativización del Pecado mediante el casuitismo ${ }^{38}$, lo que de hecho

35. Referencias literarias en las que prostituta y bruja son la misma persona, hay muchas: la misma Celestina, la madre del Buscón -"[...] que era tal su agrado, que hechizaba a cuantos la trataban"-, la negra del Diablo Cojuelo, etc.

36. Con la minuciosidad que caracterizó todo su actuar, Felipe II se ocupó de este tema, reglamentado absolutamente todo: se excluía a las mujeres casadas o con deudas, eran supervisadas diariamente por un matrimonio de reconocida moralidad, se cerraban los burdeles los días santificados, existían exámenes médicos semanales enviando a las enfermas a hospitales, se especificaron momentos de oración, vestimenta, dieta, etc. Vid. O'FAOL AIN, J. y MARTíNEZ, L. (eds.) : Not in God's Image: Women iin History from the Greeks to the Victorians, Nueva York, 1973.

37. Vid. FUSTER, J.: Heretgias revoltes i sermons, Barcelona, 1968; también, CÁTEDRA, P.: "La predicación castellana de San Vicente Ferrer", en Boletín de la Real Academia de las Buenas Letras de Barcelona, XXXIX, 1983-84, pp. 235-309.

38. Vid. sobre este sistema moral y su controversia: Guibert, J. de: La espiritualidad de la Compañía de Jesús, Santander, 1955; IPARAGUIRRE, I.: Estilo espiritual jesuítico (1540-1600), 
legitimaba determinadas prácticas inmorales -la prostitución, entre ellas- según las circunstancias en que se produjesen. No obstante, insistimos, en que no se discute entre las dos corrientes la gravedad del Pecado en la prostituta, ni su posibilidad de salvación espiritual individual, o menos aún su sistema y condiciones de vida; todas estas cuestiones no llegaron ni tan siquiera a ser planteadas; lo que de verdad se debate es la repercusión moral, secundaria según unos y fundamental según otros, que las casas públicas tenían sobre el hombre y sobre el orden establecido. Es decir, la posibilidad de poner fronteras rígidas al Pecado, o bien permitir una conducta inmoral en sí pero existente. Los defensores del "rigorismo" (dominicos, carmelitas y órdenes mendicantes), sólo fueron capaces de aplicar estrategias defensivas frente a este nueva moral catól ica triunfante y acomodaticia, usando unas argumentaciones y razonamientos ya muy gastados por el tiempo, con muy poca difusión $y$, desde luego, escaso o nulo éxito. La aceptación social y política de la prostitución como oficio, contará con la pasividad de una buena parte de la iglesia al menos hasta que esa misma sociedad y las ideas políticas que la sustentan comiencen a cambiar.

Bilbao, 1964; LONDOÑO, F.: Espíritu propio e impropio de la Compañía de Jesús (1590), Bogotá, 1963. 